\title{
Mobile crowd-sensing in the Smart City
}

\author{
Imre Lendák \\ Faculty of technical sciences, University of Novi Sad, Serbia, \\ lendak@uns.ac.rs
}

\begin{abstract}
The Smart City connects citizens in novel ways by leveraging the latest advances in information and communication technologies (ICT). Smart citizens have various ICT solutions at their disposal, which allow them to optimize their dayto-day activities in the urban environment they live and/or work. The integration of rich sensing capabilities (e.g. camera, microphone, GPS, accelerometer, barometer) in today's mobile devices allows their users to sense their urban environment in often unforeseen ways. In mobile crowd-sensing the citizens of the Smart City collect, share and jointly use services based on the sensed data, e.g. the Waze application for optimized car-based navigation, the Smart Citizen project for collecting meteorological measurements. This paper presents the current state-of-the-art and future challenges in mobile crowd-sensing in urban environments, by focusing on sensing in the following focus areas: environment, citizen collaboration, urban traffic systems, health/fitness and social networking. From each of these areas a set of representative applications (e.g. Waze, Foursquare, Ushahidi) were selected, analyzed and compared based on the following criteria: expected social and economic impact, novelty and sophistication of system architecture, sensing methods applied, motivation techniques and user privacy.
\end{abstract}

\section{Keywords}

crowd-sensing, Smart City, mobile devices, sensors

How to cite this book chapter:

Lendák, I. 2016. Mobile crowd-sensing in the Smart City. In: Capineri, C, Haklay, M, Huang, H, Antoniou, V, Kettunen, J, Ostermann, F and Purves, R. (eds.) European Handbook of Crowdsourced Geographic Information, Pp. 353-369. London: Ubiquity Press. DOI: http://dx.doi.org/10.5334/bax.z. License: CC-BY 4.0. 


\section{Introduction}

The rich sensing capabilities integrated into modern mobile devices allow their users to use them for novel, often unforeseen activities. The list of sensors integrated into the latest flagship mobile devices includes basic ones like the microphone necessary to record the user's voice and the touchscreen necessary for text input, through the also visible, one or more cameras used to record images from the users' surroundings and/or of the users themselves (i.e. selfies), as well as more obscure sensors, like the accelerometer for sensing acceleration, gyroscope for orientation, proximity for distance, compass for spatial bearing, GPS for geographic location and barometer for atmospheric pressure. The latest offerings (e.g. the Samsung Galaxy 6 Edge in early 2015) might offer personal health related sensing as well in the form of heart-rate and oxygen saturation sensing. Devices might identify their users with built-in fingerprint sensors, or via scanning and recognizing their fingerprints via their touchscreens.

The microphone, touchscreen and camera form a sufficient subset of sensors for the majority of use cases. The rest of the sensors might be used by mobile device producers to develop more user friendly behavior, e.g. automatically detecting the tilt of the mobile device with the gyroscope in order to rotate the screen accordingly.

Mobile crowd-sensing (MCS) is a relatively new discipline, in which the users of modern smart phones use the rich sensing capabilities of their devices to collect and share information while on the move, as well as to form micro-crowds around a certain crowd-sensing activity (Cardone et al. 2013). Current state and future MCS challenges were discussed in Ganti, Ye and Lei (2011), while Zambonelli (2011) dissects a more general theme, namely urban crowd-sourcing. Goodchild (2007) was one of the first identifying the crowd as a possible sensing 'tool'. The efficiency and efficacy of mobile crowd-sensing is discussed in Ma, Zhao and Yuan (2014).

The Smart City is the future city which leverages the latest advances in information and communication technologies (ICT) in order to optimize its operations and the everyday processes in which the smart citizens take part. Mobile crowd-sensing is one ICT tool which might be leveraged in Smart Cities, as it reaches the smart citizens and involve them in the optimization of the city's processes.

Crowd-sensing simulation efforts (Farkas \& Lendák 2015; Lendák \& Farkas 2015; Tanas \& Herrera-Joancomart 2013) aim to simulate crowd behavior, forecast sensing patterns and help researchers and solution developers to choose what to sense as well as to identify the minimum user threshold necessary for an application to collect sufficiently 'big' data, which the algorithms can crunch in order to produce useful information. Trustworthiness of the data sensed by the crowd is also relevant and analyzed in Tanas \& Herrera-Joancomart (2015).

Both mobile crowd-sensing and the Smart City are intriguing novel research and development domains, with numerous magazine and journal special issues 
devoted to their analysis, e.g. the August 2015, June 2013 and June 2011 issues of the IEEE Communications Magazine, the June 2013 issue of the Journal of Knowledge Economy, etc. This paper builds on those results and discusses the latest mobile crowd-sensing efforts in the Smart City setting, with a special focus on the following areas: environment, citizen collaboration, urban traffic systems, health/fitness and social networking. From each of these areas one or two representative applications were selected based on their technical sophistication and size of user base, as an easy measure of success. The solutions chosen were analyzed by trying to answer the following questions:

- How do they impact society at large (i.e. societal impact)?

-What is their expected economic impact?

-What is their system architecture like? Is it sophisticated and does it contain novel solutions?

-Which sensors and how do they employ towards reaching their goals?

- How do they motivate their users to contribute and use the application?

- How do they address the sensitive question of user privacy?

Apart from this introduction, the paper contains five sections discussing mobile crowd-sensing based applications from the above identified five focus groups. Their descriptions are followed by their comparative analysis in section seven.

\section{Urban environment}

The latest offerings in the smartphone arena come equipped with a limited set of meteorological sensors, e.g. barometer, thermometer. Apart from the obvious meteorological sensors, the integrated microphone can be used for sensing noise levels, and the camera for recording specific meteorological or other phenomena. Noise level sensing can be automated, while using the camera requires human interaction. In general, modern mobile devices are still lacking in sensing capabilities focused on collecting information about our (natural) environment. These limitations might be mitigated by purpose-built sensing hardware.

The Smart Citizen (SC) project is a mobile crowd-sensing based project whose goal is to build a platform for collecting environmental measurements in urban settings. Its website ${ }^{1}$ is pictured in Figure 1. SC is an open-source platform consisting of a hardware device (the Smart Citizen Kit), an application programming interface utilizing RESTful web services, a mobile application, a website and a web based community of volunteers, i.e. the 'crowd'. The hardware kit is equipped with sensors which measure air composition (CO and NO2), temperature, light intensity, sound levels, and humidity. It is able

\footnotetext{
${ }^{1}$ Smart Citizen project's website, https://smartcitizen.me/
} 


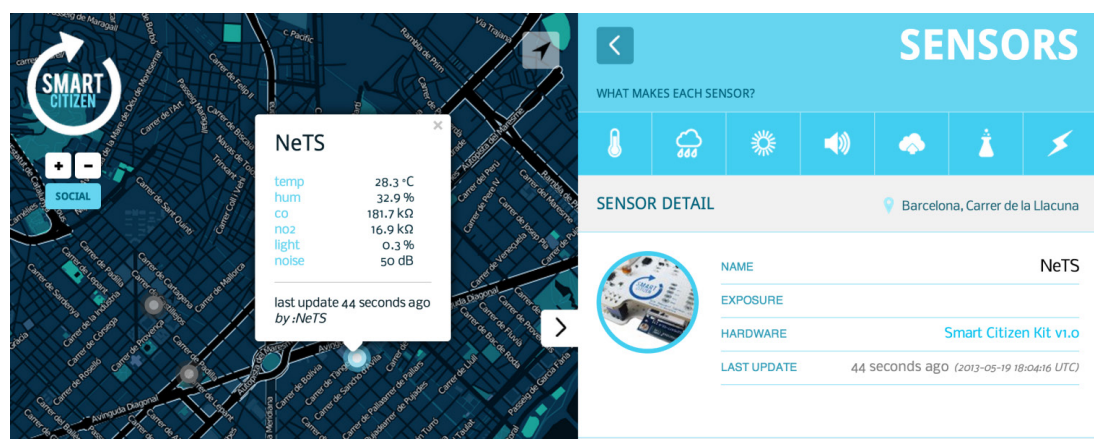

Figure 1: The Smart Citizen website.

to stream data measured by the sensors over Wi-Fi. Power to the device can be provided by a solar panel and/or battery, and it can be placed on balconies or window sills.

SC does not employ gamification or other motivation mechanisms, which might increase public interest towards this solution. It has a detailed privacy policy available via the web-based interface. Username and geographic location can be grabbed from the screen - which negatively impact smart citizen privacy. By removing the username from the web interface, the privacy level of the application could be significantly improved.

Another interesting project dealing with environmental issues in and near urban environments is Danger Maps², a crowdsourced, web-based environment monitoring solution originating from China. Its primary goal is to collect and share the locations of the various sources of pollution, e.g. garbage dumps, toxic-waste treatment facilities, oil refineries and power plants. The application is popular in China where pollution is a serious issue. It was created after its founder learned that the Shanghai apartment he bought in 2007 was near a landfill - something he wasn't informed of when negotiating the purchase. Originally, the 'old' Danger Maps contained official data and maps released by the Chinese Environmental Protection Agency, but since $2013^{3}$ the crowd is allowed to create detailed custom maps themselves via a web based interface. Danger Maps relies on social sensors (i.e. human users) as the reports are posted in textual format. The camera might be used as well for taking pictures of the pollution sources. Unfortunately, the website is available only in Chinese - or at least the author failed to find the link to an English language version.

Efforts similar to the Smart Citizen or the Danger Maps projects have significant societal impact, as they allow the crowd to collect and share information

${ }^{2}$ Danger Maps official website, http://www.epmap.org/ngo

3 Custer, C., 'Danger Maps' Invites You to Map China's Polluted Areas via New Open-Platform Maps, 2013, https://www.techinasia.com/danger-maps-invites-map-chinas-polluted-areasopenplatform-maps/ 
about both major sources of pollution and the quality of the air we breathe, which might not have been mapped otherwise. Solutions similar to Danger Maps might be especially interesting in the developing world, where laws regulate the protection of our immediate environment to a limited extent, or where modern legislation is available, but not enforced. Hopefully the 'power of the crowd' exercised via solutions similar to the projects discussed in this section, might put additional pressure on both legislative and administrative bodies in the environmental protection domain and force them to act more quickly and decisively. The immediate economic impact of these two solutions is limited at the moment, but might rise with the wider adoption of crowd-sensing, i.e. when the user bases of these projects become larger and the societies built around them gain more lobbying power.

\section{Citizen collaboration}

Crowd-sensing applications give a powerful tool into the hands of human societies, e.g. they allow citizens to reach their governments about non-essential issues they detect within their communities, like issues reported in the streets with FixMyStreet ${ }^{4}$ (see Figure 2) and similar solutions (e.g. SeeClickFix ${ }^{5}$ in the USA). These applications usually consist of a mobile application which is used for sensing and a website which displays the sensed events in near realtime (see Figure 2). FixMyStreet (FMS) is a crowd-sensing platform which can be customized for any urban area. In FMS the issue reports are linked to the reporter's email address, but FMS ensures its users that only the representatives

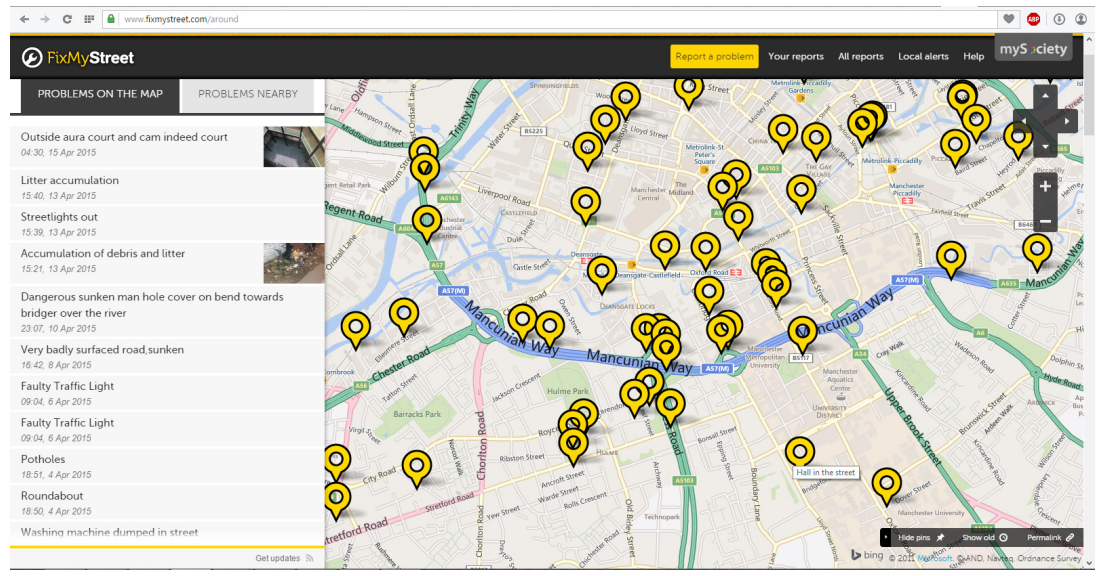

Figure 2: FixMyStreet issue reports in Manchester, UK (April 19th, 2015).

\footnotetext{
${ }^{4}$ FixMyStreet issues in Manchester, UK: https://www.fixmystreet.com/around?pc=manchester

${ }^{5}$ SeeClickFix website, http://en.seeclickfix.com
} 
of the council who will address the report, and FMS' administrative staff might be allowed to see the users' email addresses. The camera is the most important physical sensor used to take pictures of the issues the users are reporting. It does not contain motivation tools which would possibly allow it to build more effective human sensor networks, by allowing them to compete and take part in in-application games.

Crowd-sensing based citizen collaboration efforts like FixMyStreet or SeeClickFix might allow smart citizens to collaborate on issues affecting local groups in an urban environment. The loosely coupled social networks formed around these solutions might more easily obtain the attention of local administration and coax them into taking corrective action in the areas of interest, e.g. fix a pothole, or clean up an unplanned garbage dump. These solutions might have a measurable economic impact as well, mainly for local administrations, which might find out about issues sooner, fix them and spend less on paid inspectors who would travel around the urban areas and look for potholes, garbage and similar.

Crowd-sensing might allow wider collaboration during disaster relief and during political turmoil, e.g. the Ushahidi ${ }^{6}$ (Swahili for 'testimony' or 'witness') website started after Kenya's disputed presidential elections in 2007. In Ushahidi, when an event occurs a volunteer sends a brief report from a smartphone, via the Web or text message, and the software annotates it with time and location information. Such information can then be visualized and 'mined'. It relies on social sensors and on the camera, as the reports are written by people in natural language and might be accompanied by a photograph or a video. The human sensors are usually motivated by our built-in altruism, i.e. our urge to help others or contribute towards a greater good. Ushahidi itself does not contain a motivation scheme which would reward its users for sharing information. User privacy is quite important in Ushahidi, especially in countries where people might get into trouble for sharing negative views about the government or other bodies. The Ushahidi privacy policy claims that only aggregated and non-personally identifiable information is shared with third parties. The reports shared via the Ushahidi application for Android devices do not contain personally identifiable information.

Ushahidi allows the crowd of its users to report issues affecting large groups or societies, e.g. the attempted rigging of elections in Kenya ${ }^{7}$ or the 2010 earthquake in Haiti ${ }^{8}$. Therefore, it has a quite significant societal impact. Its immediate economic impact is limited, or at least it is very hard to measure.

\footnotetext{
${ }^{6}$ Ushahidi official website, http://www.ushahidi.com/

${ }^{7}$ Beyond Voting on the Ushahid official website, http://www.ushahidi.com/2015/05/21/beyondvoting-using-ushahidi-to-help-citizens-protect-their-elections/

${ }^{8}$ Ushahidi Haiti Project - Evaluation Final Report, http://www.ushahidi.com/2011/04/19/ ushahidi-haiti-project-evaluation-final-report/
} 


\section{Traffic}

Modern vehicles have rich sensing and computing capabilities, which might be used to sense and share information, e.g. they might detect when a parking spot is taken and share the information automatically. Smartphones might also detect and share certain events automatically, e.g. the Google Activity Recognition library can discern whether the device holder is walking, driving a car or running, based on the accelerometer's measurements. As it will be shown below, the most important sensors used in traffic system related crowd-sensing applications are the GPS sensor and the accelerometer.

Waze $^{9}$ is arguably the most successful crowd-sensing based application in the traffic systems domain. Its primary function is point-to-point navigation, but it performs this function with a twist: it allows drivers and other participants (e.g. co-driver) to share roadside events, e.g. road works, accidents, police presence, traffic jams. These event reports are then aggregated, shown on the map and used by the navigation algorithm, which might help drivers to avoid roadside events leading to traffic jams, e.g. a collision during rush hour. Waze is not limited to urban environments, i.e. it is not a strictly a Smart City application.

Waze consists of a mobile application used for navigation and issue reporting, a big data storage, a service for running the data analysis algorithms, and a web-based live map showing the latest events. It applies an intricate motivation scheme, which includes user levels (ranging from baby, via warrior to 'king') based on the amount of points, which might be collected through long hours of active use and issue reports, user avatars, in-app messaging and occasional inapp games. In one such game the mobile application generated Easter eggs in the streets near the driver and awarded extra points for collecting them. Waze uses the GPS sensor to calculate the current location, the camera to take pictures of the events and the accelerometer to automate certain event detections, e.g. stop-and-go traffic. Most of the events are sensed by the social sensors, i.e. the users manually annotate a roadside event by clicking on its icon in the mobile application or choosing its type from a list.

Waze links the user's account to his/her mobile phone (number) and shows an avatar onscreen (both on the mobile and in the Web based live map) at the GPS position of the user. Other nearby users are shown onscreen, not just friends, thereby allowing to learn their whereabouts based on grabbing screenshots containing their avatars. Additionally, it is possible to report non-existing roadside events, e.g. traffic jams by sending in well-formed Waze messages from a custom-built application. ${ }^{10}$ Such message fabrication attacks might be used to cause havoc in traffic systems.

\footnotetext{
9 Waze website, https://www.waze.com

10 T. Jeske, "Floating Car Data from Smartphones: What Google And Waze Know About You and How Hackers Can Control Traffic", https://media.blackhat.com/eu-13/briefings/Jeske/bh-eu13-floating-car-data-jeske-slides.pdf
} 
Apart from steering drivers clear of congestion, crowd-sensing might come in handy in solving parking problems in busy urban areas, where there are no funds to develop an advanced infrastructure of parking sensors in the streets, as done in San Francisco with the SFPark ${ }^{11}$ system, or in the City of Westminster (London) with Smart Parking. ${ }^{12}$ One such, crowd-sensing based solution, Google's OpenSpot ${ }^{13}$ tried to use the power of the crowd to sense parking related events, and based on that data provide suggestions to drivers who were looking for parking. It was cancelled in 2012 due to its limitations, mainly its inability to adapt to busy urban environments where a parking spot might remain unoccupied only for a couple of seconds, as well as for the lack of user base, i.e. the size of its user base was insufficient to make it successful. Anagog ${ }^{14}$ is a promising new player in the urban parking arena (see Figure 3). It uses Waze's data to automatically sense and share parking events - in essence it learns the habits of drivers, i.e. where and when they park their cars.

The analyzed applications address two pressing matters in the crowded urban environments of the 21st century, namely congestion and the limited availability of parking. Waze helps its users steer clear of traffic jams by utilizing the reports received from other Wazers (i.e. Waze users) who had the misfortune

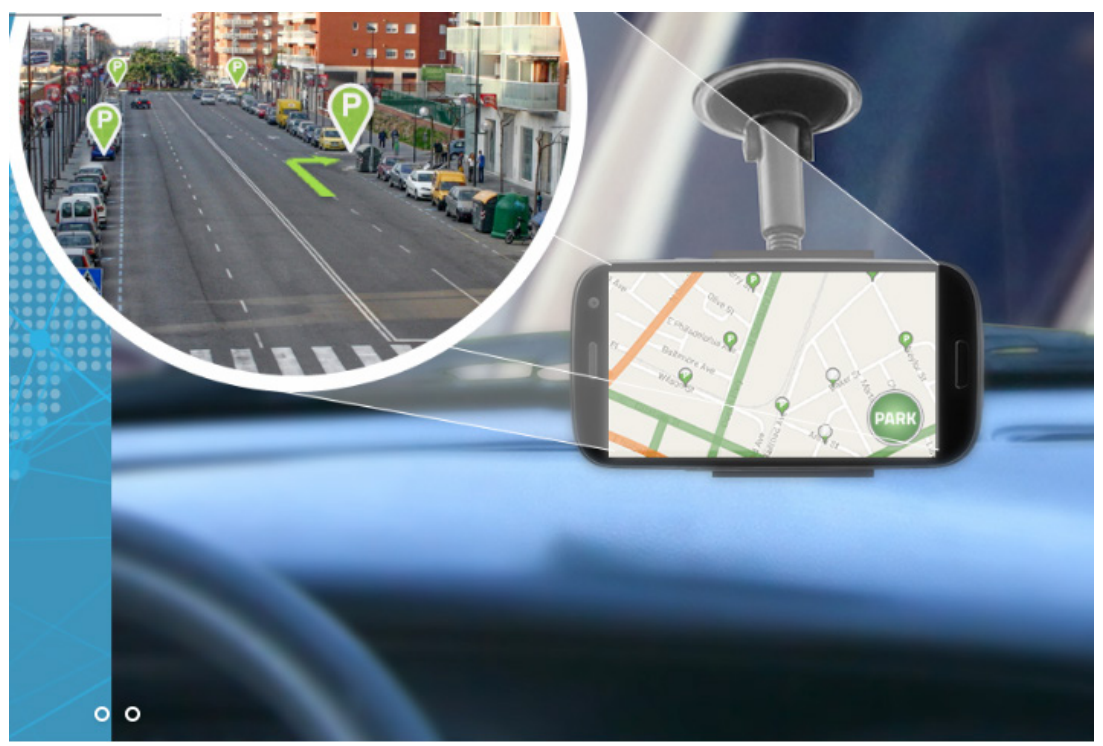

Figure 3: The Anagog parking app. ${ }^{13}$

11 SFPark website, http://sfpark.org/how-it-works/

12 Smart Parking website, http://www.smartparking.com/about-us

13 OpenSpot in the news, http://www.androidauthority.com/google-labs-open-spot-a-usefulapplication-that-no-one-uses-15186/

14 Anagog website, http://anagog.com 
of getting stuck in congestion. The parking assistance applications aim to use crowd-sensed big data in order to suggest the most likely location of an optimal parking spot, thereby reducing the time spent in 'cruising for parking', consequentially lowering petrol costs and time wasted. As both costs and time wasted might be measured in money, we conclude that these applications have a significant economic impact, especially if they reach the threshold number of active users allowing them to provide useful suggestions to the crowd.

\section{Health and fitness}

The power of the masses can contribute towards sharing information among patients suffering from specific illnesses. Apart from allowing patients to link with others who have similar health problems, the data collected and shared by patients might be used for health-care optimization, e.g. cancer survivors were planned to be brought Together in one such solution. ${ }^{15}$ Patient networking websites like PatientsLikeMe (PLM) allow individuals with certain health conditions to share and compare their symptoms and responses to the treatments they received. PLM relies on social sensors for data collection, i.e. people themselves describe their mood and physical condition, either by answering questions asked by the application, or writing textual descriptions. PLM and other similar tools might allow healthcare professionals to create more precise measurement and assessment tools based on crowd-sensed/crowd-sourced data, or might even offer early warning in case of infectious disease outbreaks. Therefore both the societal and economic impact of these solutions are significant as they can improve the prospects of sick people via mining the information shared by them and using it to develop better medicines and procedures on one hand, and potentially lowering costs on the other hand, by allowing people to learn more about their condition even before visiting a physician, and being capable of better describing how and what they feel based on the information shared by others with similar conditions.

The above listed crowd-enabled medical solutions usually have simple architectures, e.g. a website where the users might share information about their health. The users are the sensors themselves, as they describe in text how they feel and what symptoms they have. PLM applies a simple motivation scheme in which it polls its users daily in order to remind them to share information about how they feel. It also awards users with stars for sharing information about their ailments. Some news agencies reported ${ }^{16}$ that personal health information was possible to be collected from the PLM website by interested third

\footnotetext{
${ }_{15}$ Together by Medstartr, http://www.medstartr.com/projects/192-together

${ }^{16}$ CBS News, "PatiensLikeMe is more villain than victim in patient data "scraping" scandal", http://www.cbsnews.com/news/patientslikeme-is-more-villain-than-victim-in-patient-datascraping-scandal/
} 


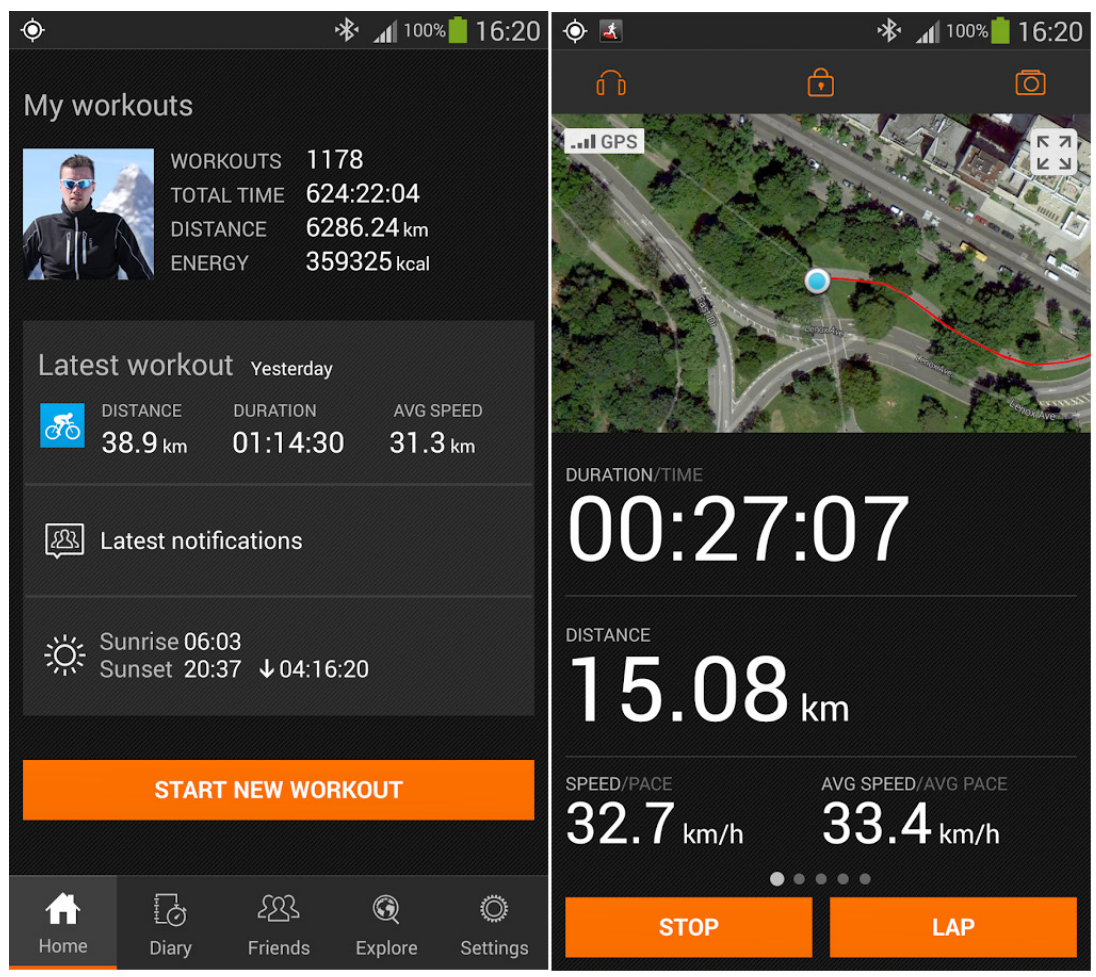

Figure 4: Sports Tracker fitness app showing fitness statistics and an on-map route.

parties, i.e. their privacy policy and its enforcement might not have been as strong as necessary.

Fitness apps (e.g. Sports Tracker ${ }^{17}$ - see Figure 4) allow their users to measure their achievements while exercising, either automatically by reading the necessary information from the built-in sensors, or by allowing users to manually enter their results. The most important sensors in fitness applications are the GPS, accelerometer and lately the heart rate monitor and oxygen saturation sensor. Fitness solutions also tend to have relatively simple architectures, consisting of a mobile app and a (cloud based) data storage, where the results of exercises are stored. Users are motivated by allowing them to post their achievements (e.g. kilometers ran) on social networks or organizing competitions with other users. Sports Tracker also has an elaborate privacy policy clearly outlining how the system uses the data collected and shared. Crowd-sensing based fitness applications can boost people's enthusiasm towards physical exercise

\footnotetext{
17 Sports Tracker, http://www.sports-tracker.com
} 
and thereby improve public health and lower the amount of funds spent on healthcare, i.e. they have a measurable societal and economic impact.

\section{Social networks}

Although Facebook posts and Twitter Tweets might contain descriptive information about our environment, events in the traffic system and even about our health and/or fitness, their primary aim is not mobile crowd-sensing. As opposed to the above named leaders in the social networking area, Foursquare (FS) (see Figure 5), the local search, discovery and recommendation app for mobiles has both social networking and sensing elements. FS takes into consideration where its users go and what they tell the application about those places, and advises other users where to go and what to visit near their current location, e.g. it might allow a user in a foreign country to find points of interest around him/her with only a smartphone and an internet connection.

The users 'sense' information about the points of interest (POIs) near their location by answering questions asked by the application. The social networking features contained in earlier versions (e.g. check-in at a location and sharing the event with friends also using FS) were factored out into a separate application named Swarm. This contributes towards privacy, as check-ins are not necessarily visible to followers. Personally identifiable information is visible in the application, as the users' full names and home town can be accessed via

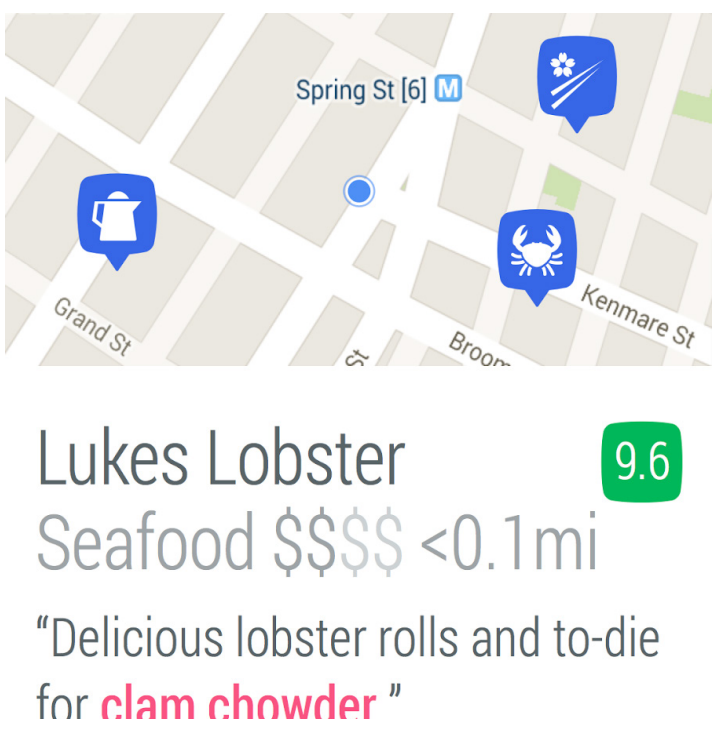

Figure 5: Foursquare location based recommendations. 
the ratings and tips they leave. Foursquare, and especially its earlier versions (prior to Swarm) employed an elaborate motivation scheme, consisting of a point system in which points were scored for each check-in, badges achieved via checking in at certain locations, and the users could become 'Mayor' of a certain venue by checking-in more often than any other user.

The architecture of Foursquare became considerably more elaborate with the introduction of Swarm, as now the solution consists of a mobile application, a backend service used for storing the big data collected from contributors and running the recommendation algorithms, as well as Swarm, which cooperates with the mobile application and adds social networking features.

Foursquare has a measurable economic impact, as it can steer visitors towards POIs (e.g. restaurant, bar) which have high ratings thereby increasing their incomes. Its societal impact is not so clear, but it can surely help its users when they are in an unknown and new environment, e.g. visiting a city in a foreign country without a local guide, by steering them towards interesting places and making them feel less out-of-place and better connected to the location they are visiting.

It is important to note that the users of other crowd-sensing based applications (e.g. Waze, FixMyStreet) might also be regarded as members of dynamic social networks, formed around certain events or activities (e.g. drivers in and around a single urban area).

\section{Comparative analysis}

The applications and solutions described in this paper were analyzed based on the following criteria: social visibility and impact, expected economic impact, sophistication of their system architectures, sensing method(s), motivation scheme and privacy level. For their comparative analysis presented in this section, from each group one or two applications were selected and marked in each of the above listed areas. The ratings were made based on publicly available material and/or the author's own experience in using the solutions. A couple of solutions were intentionally omitted from this comparative because of various reasons, e.g. Danger Maps could not be analyzed as most of the available information is in Chinese, and there was no new data available online about the Together project.

Societal impact was measured as a combination of the number of active crowd-sensors and the (expected) level of contribution of the selected application towards a greater good, e.g. improving the lives of many. The number of active crowd-sensors is not easily measured, as a varying percentage of the user base of these solutions is passive, i.e. they do not sense, just consume the services based on the sensations of others. Waze and Foursquare stand out based on the total number of their (millions of) users. Although contribution towards a greater good is even more challenging to measure, Ushahidi, FixMyStreet 
and PatientsLikeMe stand out as they might help people during disasters or other socially disruptive events, help local communities address their issues more effectively, as well as help inform patients and collect statistical information about their conditions, potentially leading to novel medical findings and procedures. Waze and Foursquare have global reach, but their mission is not as 'noble' as those of the above listed three applications. As none of the analyzed solutions have both a clear mission towards achieving a greater good, as well as millions of users globally, the author felt that they all have a mid-range impact.

Economic impact is a subjective area which is similarly challenging to measure as societal impact. The author made an attempt to compare the applications by taking into consideration the expected economic impact they 'should' make based on their primary goals. Waze and Anagog stand out as they might optimize urban traffic, via avoiding traffic jams and/or steering drivers more quickly to empty parking spots. The mid-range players are FixMyStreet, PatientsLikeMe and Foursquare. FMS allows its users to report issues in their neighborhoods to their councils, which might not know about those issues otherwise, or would need to employ people to manually identify them. PLM allows its users with various illnesses to learn about their condition from other people having similar conditions, which in turn might allow early diagnosis even before visiting a doctor, thereby lowering healthcare expenditure. FS might allow highly rated businesses to attract tourists and other visitors and thereby raise their revenue.

The novelty and complexity of system architecture was measured based on counting the total number of the following components identified during the analysis of the crowd-sensing applications: purpose-built sensing hardware, a website with live data, a mobile (sensing) application, a big data store, advanced algorithms used for analyzing the data collected and generating additional value and information, as well as social networking features. Apart from the count of various elements, another important measure was the perceived level of seamlessness of their integration into a user oriented, integrated product. Foursquare (with Swarm) and Waze are similar in not building custom sensing hardware, but having all the other components and being quite user-friendly and seamlessly integrated. Foursquare boasts an elaborate mix of information sensing and sharing mobile application combined with a social networking component (Swarm) and a powerful backend aggregating the recommendations. Waze also consists of a mobile application, a Web-based live map and backend for crunching the incoming data, creating (alternative) routes and generating various interesting in-app games with rewards to the users who participate. The Smart Citizen project on the other hand has purpose built hardware, but is lacking in the area of generating additional information and social networking features. The rest of the projects had slightly less complex system architectures, as they were either mostly web based (e.g. PLM), or seemed to be more focused on their presence on mobile devices (e.g. Sports Tracker).

The sensing capabilities of the applications were assessed based on the number of hardware sensors used by them, the sophistication of social sensor 
utilization (i.e. how well they handle the data manually entered by their users), as well as the existence of advanced signal processing algorithms applied. Waze and Sports Tracker excel in all three areas. Waze utilizes the hardware sensing features of mobile devices (e.g. GPS, accelerometer), allows its users to manually enter useful data and automates sensing via its signal processing features, e.g. the automatic detection of stop-and-go traffic. Sports Tracker also automates the collection of track length ran or cycled, and calculates the length and 'cost' (e.g. in calories) of an exercise. It also relies on the hardware sensing capabilities of mobile devices (e.g. GPS) and allows users to manually enter data about exercises whose detection it does not automate (e.g. weight lifting). The Smart Citizen project also stands out, as it is the only solution, which developed a custom-built sensing module in order to achieve its 'smart city' objective, namely to measure the various characteristics of our environment.

Crowd-sensing based solutions cannot succeed without the crowd-sensors, i.e. without their users. In today's fast-paced life it is hard to learn the habit of devoting some of our time towards collecting (i.e. sensing) data and sharing it, thereby helping others. Because of that, and in order to maintain a loyal user base, it is necessary to be innovative when trying to motivate the users. During the analysis of the crowd-sensing applications, the following motivation mechanisms were identified: financial motivation, appealing to our built-in altruistic nature (i.e. it is natural to people to try to help others), gamification, in-app games and social networking. While financial motivation was not present in the analyzed solutions, most relied on our altruism as a driving factor, with Sports Tracker being an exception as it is built for sensing tasks about a single user. The altruistic element of motivation was most prominent in Ushahidi. Gamification usually means that the application is assigning some reward (e.g. points) for each piece of data sensed, i.e. making the users feel that the use of the application is a game and thereby urging them to keep on sensing. In-app games keep the users locked to the application longer. The social networking features allow the users to somehow reach their peers. In the motivation area Waze has a dynamic points system with levels, which are obtainable after long hours of use and contributing information, as well as in-app games and messaging. It also plays on the natural altruism of its users, as it allows them to help others via marking a police presence or speed control. Foursquare also had a points-based system, badges awarded for certain check-ins, social networking layer for staying in contact with friends and it allowed users to become 'mayors' of the places they visited more often than others. This latter might be regarded as a hybrid form of a gamification feature and an in-app game. PatientsLikeMe has a modest motivation scheme consisting of daily polling in which it asks its users to share information about their health. Apart from that it also awards up to three stars for sharing specific information.

The following privacy aspects of the crowd-sensing solutions were taken into consideration: the amount of personally identifiable information shown on screen to other users, the existence of news reports or other proof about 
privacy breaches and the sophistication of techniques used to ensure the privacy of the crowd-sensors. In FixMyStreet the personal information collected is limited, it is not shown on screen and, according to the privacy policy, it is shared only with system administrators and council members who might be contacted to address the issue reports sent via the application. Ushahidi anonymizes the contributions of its users, there were no reports about privacy breaches at the moment of writing, and it also has an elaborate privacy policy. Sports Tracker also applies the privacy components and there were no known privacy breaches. The Smart Citizen project and Waze apply the above techniques, but show both the username and the location of the sensors on screen, which might allow malevolent third parties to misuse that information. The rest of the analyzed applications also employ techniques which raise the level of user privacy, but they either do not completely hide personally identifiable information (e.g. full name and address visible in Foursquare), or there were news reports about some form of privacy breaches (e.g. PLM).

Table 1 contains a comparative overview of the analyzed crowd-sensing based solutions. The following three types of marks were assigned based on their analysis laid out above:

- Leader/High impact - the solution applies the majority of the available techniques in the subject domain or has the highest expected social/economic impact.

- Mid-range capabilities/impact - the solution applies some of the techniques relevant in the area or has medium societal/economic impact.

- Limited capabilities/low impact - the solution has limited capabilities or its societal/economic impact is marginal.

Essentially, the analyzed applications are quite different and their comparison was done by analyzing some of their common, key aspects. The author did not

\begin{tabular}{|l|c|c|c|c|c|c|}
\hline \multicolumn{1}{|c|}{ App/Solution } & $\begin{array}{c}\text { Societal } \\
\text { impact }\end{array}$ & $\begin{array}{c}\text { Economic } \\
\text { impact }\end{array}$ & Architect. & Sensing & Motiv. & Privacy \\
\hline Smart Citizen & $\bullet \bullet$ & $\bullet$ & $\bullet$ & $\bullet$ & $\bullet$ & $\bullet$ \\
\hline FixMyStreet & $\bullet$ & $\bullet$ & $\bullet$ & $\bullet$ & $\bullet$ & $\bullet \bullet$ \\
\hline Ushahidi & $\bullet$ & $\bullet$ & $\bullet$ & $\bullet$ & $\bullet$ & $\bullet \bullet$ \\
\hline Waze + Anagog & $\bullet$ & $\bullet \bullet$ & $\bullet$ & $\bullet \bullet$ & $\bullet \bullet$ & $\bullet$ \\
\hline PatientsLikeMe & $\bullet$ & $\bullet$ & $\bullet$ & $\bullet$ & $\bullet \bullet$ & $\bullet$ \\
\hline Sports Tracker & $\bullet$ & $\bullet$ & $\bullet$ & $\bullet \bullet$ & $\bullet$ & $\bullet \bullet$ \\
\hline Foursquare & $\bullet$ & $\bullet$ & $\bullet$ & $\bullet$ & $\bullet \bullet$ & $\bullet$ \\
\hline
\end{tabular}

Table 1: Comparative analysis of crowd-sensing based applications $(\bullet \bullet-$-leader/ high impact, $\bullet \bullet-$ mid-range capabilities/impact, $\bullet$ - limited capabilities/ impact). 
attempt to directly compare the solutions based on the number of 'points' they were awarded in Table 1.

The author plans to extend the initial results presented in this section as part of his future research, by including a more detailed measurement of the number of total and active users of each solution, adding a more detailed econometric analysis of the diverse solutions discussed, as well as by identifying and including them additional solutions in each group and localizing the comparisons inside each group, thereby avoiding directly comparing health and traffic apps to each other.

\section{Summary}

This paper contains a comparative analysis mobile crowd-sensing solutions in the Smart City environment. The analysis was focused on crowd-sensing in the following focus areas: environment, citizen collaboration, urban traffic systems, health/fitness and social networking. From each of these focus areas up to two successful applications were selected and analyzed. The applications were analyzed (e.g. Waze, Foursquare, Ushahidi, the Smart Citizen project, PatientsLikeMe) by a compound comparison criteria consisting of the following elements: societal and economic impact, sophistication of system architecture, novel methods of sensor use, motivation scheme and steps taken towards ensuring user privacy.

As a continuation of this work, the author intends to identify additional crowdsensing-based applications in the Smart City ecosystem and do a more detailed comparative analysis, especially in the areas which might be objectively measured, e.g. number of total vs active users, economic impact via econometrics, etc. Apart from that, the author also plans to analyze crowd-sensing application use through space and time and find patterns leading to success or failure.

\section{Acknowledgment}

This work was partially supported the Hungarian National Academy of Sciences under grant Domus 105/6864/HTMT.

\section{References}

Cardone, G., Foschini, L., Bellavista, P., Corradi, A., Borcea, C., Talasila, M., \& Curtmola, R. 2013. Fostering ParticipAction in smart cities: a geo-social platform. IEEE Communication Magazine, 51(6): 112-119.

Farkas, K., \& Lendák, I. 2015. Simulation Environment for Investigating Crowd-sensing Based Urban Parking. In: Models and Technologies for Intel- 
ligent Transportation Systems (MT-ITS 2015), Budapest, Hungary, June 2015, pp. 320-327.

Ganti, R. K., Ye F., \& Lei, H. 2011. Mobile crowdsensing: current state and future challenges. IEEE Communications Magazine, 49(11): 32-39.

Goodchild, M. F. 2007. Citizens as sensors: the world of volunteered geography. GeoJournal, 69(4): 211-221.

Lendák, I., \& Farkas, K. 2015. Evaluation of simulation engines for crowdsensing activities. In: 3rd International Conference \& Workshop on Mechatronics in Practice and Education (Mechedu 2015). Subotica, Serbia, May 2015, pp. 82-87.

Ma, H., Zhao, D., \& Yuan P. 2014. Opportunities in mobile crowd sensing. IEEE Communications Magazine, 52(8): 29-35.

Tanas, C., \& Herrera-Joancomart, J. 2014. Crowdsensing simulation using ns-3. Citizen in Sensor Networks, Lecture Notes in Computer Science, pp. 47-58.

Tanas, C., \& Herrera-Joancomart, J. 2015. When users become sensors: Can we trust their readings? International Journal of Communication Systems, 28(4): 601-614.

Zambonelli, F. 2011. Pervasive urban crowdsourcing: visions and challenge. In: 2011 IEEE International Conference on Pervasive Computing and Communications Workshops (PERCOM Workshops). Seattle, USA, March 2011, pp. 578-583. 